\title{
Synthesis and Evaluation of Nematicidal Activity of Compounds Derived from Norbornadiene
}

\author{
Anni C. S. Gomes, ${ }^{a}$ Antonio J. Demuner, ${ }^{\circledR} * a$ Elson S. Alvarenga, ${ }^{\circledR a}$ João P. E. Gondim, ${ }^{b}$ \\ Andressa R. Fonseca, ${ }^{b}$ Dalila S. Buonicontro, ${ }^{b}$ Eduardo J. Pilau ${ }^{\circledR c}$ and Evandro Silva ${ }^{\circledR c}$ \\ a Departamento de Química, Universidade Federal de Viçosa, 36570-900 Viçosa-MG, Brazil \\ ${ }^{b}$ Departamento de Fitopatologia, Universidade Federal de Viçosa, 36570-900 Viçosa-MG, Brazil \\ 'Departamento de Química, Universidade Estadual de Maringá, 87020-900 Maringá-PR, Brazil
}

\begin{abstract}
Nematode is a major problem in agriculture, the effective way to control this pest is through chemical control, but the efficient molecules present in the market have great toxicity to mammals and birds. Thus, there is a constant demand for the development of new nematicide molecules. This study describes the synthesis of 21 novel esters derived from norbornadiene and evaluation of their potential activity against Meloidogyne javanica. The esters 5-25 presented 50\% mortality of the nematodes. The acid $\mathbf{3}$ and the ester $\mathbf{4}$ caused 96 and $93 \%$ mortality of the nematodes, respectively. The lethal concentration to kill $50 \%$ of the nematodes $\left(\mathrm{LD}_{50}\right)$ were determined as being $11.8 \mu \mathrm{g} \mathrm{mL}{ }^{-1}$ for the acid $\mathbf{3}$ and $99.4 \mu \mathrm{g} \mathrm{mL}^{-1}$ for the ester $\mathbf{4}$. According to the results obtained it is believed that polarity seems to be a key factor for the higher activity of compound $\mathbf{3}$ compared to structurally similar ester derivatives.
\end{abstract}

Keywords: Meloidogyne javanica, Diels-Alder, bicyclo[2.2.1]hepta-2,5-diene-2,3-dicarboxylic acid, but-2-ynedioic acid

\section{Introduction}

Synthetic organic chemists are always involved in the production of new substances that have effective biological activity and are less toxic to humans and the environment.

Among the different synthetic strategies, the $[4+2]$ cycloaddition, commonly known as Diels-Alder reaction, is gaining more and more prominence..$^{1-4}$ Several products synthesized by this reaction have effective application in our daily lives, and we can mention the case of the agrochemical Captan, which presents as key step the Diels-Alder reaction. With this reaction it is possible to obtain bicycles that are very active in the control of different biological agents. ${ }^{5-9}$

A bicyclic compound that has been excelling in recent years is the norbornadiene, a homoalkyl diene system compound, ${ }^{1,4}$ of great scientific interest in chemistry due to its high symmetry, unusual structure besides a high reactivity. ${ }^{10,11}$ An area that can be mentioned is its use as a sensitizer in energy production processes..$^{12-15} \mathrm{In}$ addition, several reports involving theoretical chemistry for the analysis of the structure and bonds of this kind of

*e-mail: ademuner@ufv.br compounds are described in literature. ${ }^{16-18}$ However, there are not reports of norbornadiene and its derivatives as an agrochemical yet.

A problem in agriculture that has high economic impact are the nematodes known as an economically harmful group of parasites. ${ }^{19}$ The main species that causes damage is the Meloidogyne, which causes root damage that directly affects the water and nutrients absorption necessary for the good development of the plants. ${ }^{20}$ Nematodes are estimated to generate a projected annual loss of $12.3 \%$ (\$ 157 billion) worldwide. ${ }^{21}$ Among the main procedures for accomplishing the management of cultures against attack by nematodes, chemical control is the most efficient and fast method. ${ }^{22}$ Due to restrictions of some nematicides it is necessary to search for new molecules to supply the demand in this segment.

As part of a program directed towards the synthesis of bioactive compounds, ${ }^{23-26}$ we were interested in preparing substances with potential agrochemical activities. Lactones and amides were shown to present diverse biological activities. Therefore, a study was conducted to verify the potential of bicyclic dicarboxylic acid and bicyclic dialkyl dicarboxylate derivatives as nematicides. 
For this purpose, 21 bicyclic esters were synthesized from a norbornadiene dicarboxylic acid (3) via reaction with alkyl and benzyl bromides (5-25). The methyl ester (4) was synthesized by reaction of the norbornadiene dicarboxylic acid with methanol catalyzed by sulfuric acid. The compounds synthesized were evaluated against Meloidogyne javanica.

\section{Experimental}

\section{General}

Acetylenedicarboxylic acid monopotassium salt (potassium 3-carboxypropiolate) and dicyclopentadiene (454338-1 kg) were acquired from Sigma-Aldrich (A15401, St. Louis, United States). Infrared (IR) spectra were performed on a FT-IR Varian 660 equipped with GladiATR (attenuated total reflection accessory, Varian, Palo Alto, CA, USA). Nuclear magnetic resonance (NMR) spectra were recorded on a Varian Mercury spectrometer $(300 \mathrm{MHz})$ and Bruker DPX 200-Avance spectrometer (200 MHz). The solvent employed was deuterated chloroform, and the signal of the hydrogen of the residual $\mathrm{CHCl}_{3}$ was used as the reference $(\delta 7.26)$ in the ${ }^{1} \mathrm{H}$ NMR. The signal of the carbon of $\mathrm{CDCl}_{3}$ was used as the reference $(\delta 77)$ in the ${ }^{13} \mathrm{C}$ NMR. High resolution mass spectra (HRMS) analysis were recorded using a quadrupole time-of-flight mass spectrometer (Impact II, Bruker Daltonics Corporation, Germany) equipped with an electrospray ionization (ESI) source. Samples were directly injected into the electrospray ionization source using a syringe pump (KDS Legato 100 , KD Scientific, Holliston, MA, USA) at a flow rate of $10 \mu \mathrm{L} \mathrm{min}^{-1}$. The capillary voltage was operated in positive ion mode, set at $4000 \mathrm{~V}$ with an end plate offset potential of $-500 \mathrm{~V}$. The dry gas parameters were set to $8 \mu \mathrm{L} \mathrm{min}^{-1}$ at $180^{\circ} \mathrm{C}$ with a nebulization gas pressure of 0.4 bar. Data were collected in the range of $\mathrm{m} / \mathrm{z} 50$ to 1000 in continuous acquisition mode. The mass spectra were obtained in the DataAnalysis program 4.3 (Bruker Daltonics Corporation, Germany). The quality (purity) of the compounds was assessed by the ${ }^{1} \mathrm{H}$ and ${ }^{13} \mathrm{C}$ NMR spectroscopy and their identity confirmed by the combined interpretation of NMR, IR, and mass spectrometry (HRMS).

\section{Synthesis of bicyclo[2.2.1] hepta-2,5-diene-2,3-dicarboxylic} acid (3)

Acetylenedicarboxylic acid monopotassium salt $(2.00 \mathrm{~g})$, concentrated hydrochloric acid $(2.00 \mathrm{~mL})$, cyclopentadiene $(4.00 \mathrm{~mL}$, freshly distilled) and water $(10.00 \mathrm{~mL})$ in a round bottomed flask was stirred at $25{ }^{\circ} \mathrm{C}$ for $4 \mathrm{~h}$. The reaction mixture was extracted with chloroform $(3 \times 15 \mathrm{~mL})$ and the organic solvent was removed under vacuum. The yellow residue was washed with hexane $(30 \mathrm{~mL})$ to afford the desired product bicyclo[2.2.1] hepta-2,5-diene-2,3-dicarboxylic acid (3). ${ }^{26}$ This bicycle was used as a starting material for the synthesis of all esters derivatives.

Yellow solid; yield 79\%; $\mathrm{mp} 160-161{ }^{\circ} \mathrm{C} ; \mathrm{Rf}=0.15$ (hexane/ethyl acetate 1:1 v/v); IR (ATR) $\mathrm{v} / \mathrm{cm}^{-1} 3370$ 2710, 2983, 2952, 2877, 1693, 1619, 1577, 1442, 1220, 696; ${ }^{1} \mathrm{H}$ NMR $\left(200 \mathrm{MHz}, \mathrm{CDCl}_{3}\right) \delta$ 2.12-2.19 (m, $1 \mathrm{H}$, H7a), 2.28-2.31 (m, 1H, H7b), 4.20-4.21 (m, 2H, H1, H4), 6.93-6.94 (m, 2H, H5, H6), 11.86 (s, 1H, H9); ${ }^{13} \mathrm{C}$ NMR $\left(50 \mathrm{MHz}, \mathrm{CDCl}_{3}\right) \delta 54.3(\mathrm{C} 1, \mathrm{C} 4), 73.2$ (C7), 142.3 (C5, C6), 158.1 (C2, C3), 167.4 (C8); HRMS (ESI) $\mathrm{m} / z$, calcd. for $\mathrm{C}_{9} \mathrm{H}_{8} \mathrm{NaO}_{4}[\mathrm{M}+\mathrm{Na}]^{+}$: 203.0315, found: 203.0312.

General procedure for syntheses of the esters derived from bicyclo[2.2.1] hepta-2,5-diene-2,3-dicarboxylic acid (5-25)

A mixture of compound $3(0.180 \mathrm{~g}, 1.00 \mathrm{mmol})$, $N, N$-dimethylacetamide (DMA, $5.00 \mathrm{~mL}$ ), and $\mathrm{K}_{2} \mathrm{CO}_{3}$ $(0.690 \mathrm{~g}, 6.00 \mathrm{mmol})$ in a round bottomed flask was stirred at room temperature for $1 \mathrm{~h}$. Benzyl bromide $(2.20 \mathrm{mmol})$ was added to the reaction mixture and stirred for $18 \mathrm{~h}$ at $25^{\circ} \mathrm{C}$. When alkyl bromide (instead of benzyl bromide) was used, the reaction mixture was heated at $70{ }^{\circ} \mathrm{C}$ for $24 \mathrm{~h}$. The reaction was quenched with $0.630 \mathrm{~mol} \mathrm{~L}^{-1}$ hydrochloric acid $(30 \mathrm{~mL})$ and the mixture was extracted with ethyl acetate $(3 \times 20 \mathrm{~mL})$. The combined organic phases was dried with anhydrous sodium sulfate, filtered and concentrated under reduced pressure. The residue was purified by silica-gel column chromatography using mixtures of hexane/ethyl acetate as described below.

Diisopropyl bicyclo[2.2.1]hepta-2,5-diene-2,3-dicarboxylate (5)

Yellow oil; yield 74\%; Rf $=0.47$ (hexane/ethyl acetate $4: 1 \mathrm{v} / \mathrm{v}$ ); IR (ATR) $\mathrm{v} / \mathrm{cm}^{-1} 2977,2937,1699,1624,1261$, 1093, 705; ${ }^{1} \mathrm{H}$ NMR $\left(200 \mathrm{MHz}, \mathrm{CDCl}_{3}\right) \delta 1.28-1.31(\mathrm{~m}$, $\left.12 \mathrm{H}, 4 \times \mathrm{CH}_{3}\right), 2.04-2.09(\mathrm{~m}, 1 \mathrm{H}, \mathrm{H} 7 \mathrm{a}), 2.25-2.30(\mathrm{~m}, 1 \mathrm{H}$, H7b), 3.88-3.92 (m, 2H, H1, H4), 5.00-5.19 (m, 2H, H9), 6.90-6.92 (m, 2H, H5, H6); ${ }^{13} \mathrm{C}$ NMR (50 MHz, $\mathrm{CDCl}_{3}$ ) $\delta 21.8\left(4 \times \underline{\mathrm{CH}}_{3}\right), 53.4(\mathrm{C} 1, \mathrm{C} 4), 68.6(\mathrm{C} 9), 72.6(\mathrm{C} 7)$, 142.4 (C5, C6), 152.0 (C2, C3), 164.9 (C8); HRMS (ESI) $m / z$, calcd. for $\mathrm{C}_{15} \mathrm{H}_{20} \mathrm{NaO}_{4}[\mathrm{M}+\mathrm{Na}]^{+}: 287.1254$, found: 287.1251 .

Di(prop-2-yn-1-yl) bicyclo[2.2.1]hepta-2,5-diene2,3-dicarboxylate (6)

Yellow oil; yield $65 \% ; \mathrm{Rf}=0.38$ (hexane/ethyl acetate $3: 1 \mathrm{v} / \mathrm{v})$; IR (ATR) $\mathrm{v} / \mathrm{cm}^{-1} 3294,2951,2883,1707,1618$, 
1232, 714; ${ }^{1} \mathrm{H}$ NMR (200 MHz, $\left.\mathrm{CDCl}_{3}\right) \delta 2.08-2.13(\mathrm{~m}, 1 \mathrm{H}$, H7a), 2.27-2.32 (m, 1H, H7b), 2.50 (t, 2H, J $2.5 \mathrm{~Hz}, \mathrm{H} 11$ ), 3.95-3.99 (m, 2H, H1, H4), 4.77 (d, 4H, J $2.5 \mathrm{~Hz}, \mathrm{H} 9$ ), 6.90-6.93 (m, 2H, H5, H6); ${ }^{13} \mathrm{C}$ NMR (50 MHz, $\mathrm{CDCl}_{3}$ ) $\delta 52.6$ (C1, C4), 53.7 (C9), 73.3 (C7), 75.4 (C11), 77.4 (C10), 142.5 (C5, C6), 152.7 (C2, C3), 164.1 (C8); HRMS (ESI) $\mathrm{m} / z$, calcd. for $\mathrm{C}_{9} \mathrm{H}_{8} \mathrm{NaO}_{4}[\mathrm{M}+\mathrm{Na}]^{+}:$203.0315, found: 203.0312 .

Dipentyl bicyclo[2.2.1]hepta-2,5-diene-2,3-dicarboxylate (7)

Yellow oil; yield 50\%; $\mathrm{Rf}=0.48$ (hexane/ethyl acetate 3.9:0.1 v/v); IR (ATR) v / $\mathrm{cm}^{-1} 2958,2862,1708,1630$, 1461, 1228, 1099, 708; ${ }^{1} \mathrm{H}$ NMR $\left(200 \mathrm{MHz}, \mathrm{CDCl}_{3}\right)$ $\delta$ 0.86-0.92 (m, 6H, $\left.2 \times \mathrm{C}_{3}\right), 1.28-1.35(\mathrm{~m}, 8 \mathrm{H}, \mathrm{H} 11$, H12), 1.58-1.68 (m, 4H, H10), 2.04-2.08 (m, 1H, H7a), 2.25-2.29 (m, 1H, H7b), 3.89-3.91 (m, 2H, H1, H4), 4.14 (t, 4H, J 6.8 Hz, H9), 6.89 (t, 2H, J $2.0 \mathrm{~Hz}, \mathrm{H} 5, \mathrm{H} 6$ ); ${ }^{13} \mathrm{C}$ NMR $\left(50 \mathrm{MHz}, \mathrm{CDCl}_{3}\right) \delta 13.9(\mathrm{C} 13), 22.3$ (C12), 28.0 (C11), 28.2 (C10), 53.4 (C1, C4), 65.1 (C9), 72.7 (C7), 142.4 (C5, C6), 152.1 (C2, C3), 165.3 (C8); HRMS (ESI) $m / z$, calcd. for $\mathrm{C}_{19} \mathrm{H}_{28} \mathrm{NaO}_{4}[\mathrm{M}+\mathrm{Na}]^{+}: 343.1868$, found: 343.1880 .

Dihexyl bicyclo[2.2.1]hepta-2,5-diene-2,3-dicarboxylate (8)

Colorless oil; yield 55\%; $\mathrm{Rf}=0.52$ (hexane/ethyl acetate 3.9:0.1 v/v); IR (ATR) $\mathrm{v} / \mathrm{cm}^{-1} 2937,2854,1710$, $1630,1460,1253,1228,712 ;{ }^{1} \mathrm{H}$ NMR $\left(300 \mathrm{MHz}, \mathrm{CDCl}_{3}\right)$ $\delta$ 0.86-0.90 (m, $\left.6 \mathrm{H}, 2 \times \mathrm{C}_{3}\right), 1.27-1.39\left(\mathrm{~m}, 12 \mathrm{H}, 3 \times \mathrm{C}_{2}\right)$, 1.60-1.69 (m, 4H, H10), 2.07 (dt, 1H, J 6.7, 1.4 Hz, H7a), 2.27 (dt, 1H, J 6.7, $1.5 \mathrm{~Hz}, \mathrm{H} 7 \mathrm{~b}$ ), 3.90-3.92 (m, H1, H4), 4.14 (t, 4H, J 6.8 Hz, H9), 6.90 (t, 2H, J 2.0 Hz, H5, H6); ${ }^{13} \mathrm{C}$ NMR $\left(75 \mathrm{MHz}, \mathrm{CDCl}_{3}\right) \delta 14.0(\mathrm{C} 14), 22.5$ (C13), 25.5 (C12), 28.5 (C11), 31.4 (C10), 53.4 (C1, C4), 65.2 (C9), 72.7 (C7), 142.4 (C5, C6), 152.1 (C2, C3), 165.2 (C8); HRMS (ESI) $m / z$, calcd. for $\mathrm{C}_{21} \mathrm{H}_{32} \mathrm{NaO}_{4}[\mathrm{M}+\mathrm{Na}]^{+}$: 371.2193, found: 371.2190 .

Dihepthyl bicyclo[2.2.1]hepta-2,5-diene-2,3-dicarboxylate (9)

Yellow oil; yield 56\%; $\mathrm{Rf}=0.48$ (hexane/ethyl acetate 3.5:0.5 v/v); IR (ATR) v / cm ${ }^{-1} 2923,2960,1712,1630$, $1248,1009,712 ;{ }^{1} \mathrm{H}$ NMR (300 MHz, $\left.\mathrm{CDCl}_{3}\right) \delta 0.85-0.90$ (m, 6H, $\left.2 \times \underline{\mathrm{CH}}_{3}\right), 1.27-1.31\left(\mathrm{~m}, 16 \mathrm{H}, 8 \times \mathrm{C}_{2}\right), 1.60-1.70$ (m, 4H, H10), 2.06 (dt, 1H, J 6.7, 1.5 Hz, H7a), 2.28 (dt, $1 \mathrm{H}, J 6.7,1.5 \mathrm{~Hz}, \mathrm{H} 7 \mathrm{~b}$ ), 3.90-3.92 (m, 2H, H1, H4), 4.14 (t, $4 \mathrm{H}, J 6.8 \mathrm{~Hz}, \mathrm{H} 9), 6.90$ (t, 2H, J $2.0 \mathrm{~Hz}, \mathrm{H} 5, \mathrm{H} 6) ;{ }^{13} \mathrm{C} \mathrm{NMR}$ $\left(75 \mathrm{MHz}, \mathrm{CDCl}_{3}\right) \delta 14.0(\mathrm{C} 15), 22.5(\mathrm{C} 14), 25.8(\mathrm{C} 11)$, 28.5 (C12), 28.9 (C10), 31.8 (C13), 53.4 (C1, C4), 65.1 (C9), 72.8 (C7), 142.4 (C5, C6), 152.0 (C2, C3), 165.3 (C8); HRMS (ESI) $m / z$, calcd. for $\mathrm{C}_{23} \mathrm{H}_{36} \mathrm{NaO}_{4}[\mathrm{M}+\mathrm{Na}]^{+}$: 399.2506, found: 399.2495 .
Dioctyl bicyclo[2.2.1]hepta-2,5-diene-2,3-dicarboxylate (10)

Colorless oil; yield 58\%; Rf $=0.41$ (hexane/ethyl acetate 3.9:0.1 v/v); IR (ATR) $v / \mathrm{cm}^{-1} 2929,2858,1712$, $1630,1228,712 ;{ }^{1} \mathrm{H}$ NMR $\left(300 \mathrm{MHz}, \mathrm{CDCl}_{3}\right) \delta$ 0.85-0.89 $\left(\mathrm{m}, 6 \mathrm{H}, 2 \times \mathrm{C}_{3}\right), 1.25-1.27\left(\mathrm{~m}, 20 \mathrm{H}, 10 \times \mathrm{C}_{2}\right), 1.60-$ 1.69 (m, 4H, H10), 2.07 (dt, 1H, J 6.7, $1.5 \mathrm{~Hz}, \mathrm{H} 7 \mathrm{a}$ ), 2.27 (dt, 1H, J 6.7, 1.6 Hz, H7b), 3.90-3.92 (m, 2H, H1, H4), 4.14 (t, 4H, J 6.8 Hz, H9), 6.90 (t, 2H, J 2.0 Hz, H5, H6); ${ }^{13} \mathrm{C} \mathrm{NMR}\left(75 \mathrm{MHz}, \mathrm{CDCl}_{3}\right.$ ) $\delta 14.2$ (C16), 22.8 (C15), 26.0 (C13), 28.7 (C12), 29.3 (C11), 29.4 (C10), 31.9 (C14), 53.6 (C1, C4), 65.3 (C9), 72.9 (C7), 142.5 (C5, C6), 152.2 (C2, C3), 165.4 (C8); HRMS (ESI) $m / z$, calcd. for $\mathrm{C}_{25} \mathrm{H}_{40} \mathrm{NaO}_{4}$ $[\mathrm{M}+\mathrm{Na}]^{+}:$427.2819, found: 427.2819 .

Didecyl bicyclo[2.2.1] hepta-2,5-diene-2,3-dicarboxylate (11)

Colorless oil; yield 59\%; Rf $=0.82$ (hexane/ethyl acetate 3.9:0.1 v/v); IR (ATR) $v / \mathrm{cm}^{-1} 2918,2856,1712$, 1624, 1248, 1234, 712; ${ }^{1} \mathrm{H}$ NMR (300 MHz, $\mathrm{CDCl}_{3}$ ) $\delta$ 0.85-0.89 (m, $\left.6 \mathrm{H}, 2 \times \mathrm{C}_{3}\right), 1.26-1.36(\mathrm{~m}, 28 \mathrm{H}$, $\left.14 \times \mathrm{C}_{2}\right), 1.60-1.67$ (m, 4H, H10), 2.07 (dt, 1H, J 6.8, $1.5 \mathrm{~Hz}, \mathrm{H} 7 \mathrm{a}$ ), 2.27 (dt, 1H, J 6.8, 1.6 Hz, H7b), 3.90-3.92 (m, 2H, H1, H4), 4.14 (t, 4H, J 6.8 Hz, H9), 6.90 (t, 2H, $J 2.0 \mathrm{~Hz}, \mathrm{H} 5, \mathrm{H} 6) ;{ }^{13} \mathrm{C} \mathrm{NMR}\left(75 \mathrm{MHz}, \mathrm{CDCl}_{3}\right) \delta 14.1$ (C18), 22.6 (C17), 25.9 (C11), 28.5 (C12), 29.3 (C13, C14), 29.5 (C15, C10), 31.9 (C16), 53.4 (C1, C4), 65.2 (C9), 72.7 (C7), 142.4 (C5, C6), 152.1 (C2, C3), 165.3 (C8); HRMS (ESI) $\mathrm{m} / z$, calcd. for $\mathrm{C}_{29} \mathrm{H}_{48} \mathrm{NaO}_{4}[\mathrm{M}+\mathrm{Na}]^{+}$: 483.3445, found: 483.3438 .

Didodecyl bicyclo[2.2.1]hepta-2,5-diene-2,3-dicarboxylate (12)

Yellow oil; yield 59\%; Rf $=0.46$ (hexane/ethyl acetate 3.9:0.1 v/v); IR (ATR) v / cm ${ }^{-1} 2923,2854,1707,1624$, 1234, 708; ' $\mathrm{H}$ NMR $\left(300 \mathrm{MHz}, \mathrm{CDCl}_{3}\right) \delta 0.85-0.90(\mathrm{~m}$, $\left.6 \mathrm{H}, 2 \times \mathrm{CH}_{3}\right), 1.26-1.34\left(\mathrm{~m}, 36 \mathrm{H}, 18 \times \mathrm{CH}_{2}\right), 1.60-1.70(\mathrm{~m}$, 4H, H10), 2.07 (dt, 1H, J 6.8, $1.6 \mathrm{~Hz}, \mathrm{H} 7 \mathrm{a}), 2.27$ (dt, 1H, $J 6.8,1.7 \mathrm{~Hz}, \mathrm{H} 7 \mathrm{~b}$ ), 3.90-3.92 (m, 2H, H1, H4), 4.14 (t, 4H, $J 6.8 \mathrm{~Hz}, \mathrm{H} 9), 6.91$ (t, 2H, J $2.0 \mathrm{~Hz}, \mathrm{H} 5, \mathrm{H} 6) ;{ }^{13} \mathrm{C} \mathrm{NMR}$ (75 MHz, $\mathrm{CDCl}_{3}$ ) $\delta 14.1$ (C20), 22.7 (C19), 25.9 (C11), 28.5 (C12), 29.3 (C13, C14), 29.5 (C15), 29.6 (C10, C16, C17), 31.9 (C18), 53.5 (C1, C4), 65.2 (C9), 72.8 (C7), 142.4 (C5, C6), 152.1 (C2, C3), 165.3 (C8); HRMS (ESI) $\mathrm{m} / 2$, calcd. for $\mathrm{C}_{33} \mathrm{H}_{56} \mathrm{NaO}_{4}[\mathrm{M}+\mathrm{Na}]^{+}$: 539.4071, found: 539.4066 .

Dibenzyl bicyclo[2.2.1]hepta-2,5-diene-2,3-dicarboxylate (13)

Yellow oil; yield $60 \% ; \mathrm{Rf}=0.38$ (hexane/ethyl acetate, 2.9:0.1 v/v); IR (ATR) v / cm ${ }^{-1} 3035,2950,2870,1709$, 1626, 1224, 696; ${ }^{1} \mathrm{H}$ NMR $\left(300 \mathrm{MHz}, \mathrm{CDCl}_{3}\right) \delta 2.10$ (dt, $1 \mathrm{H}, J 6.8,1.5 \mathrm{~Hz}, \mathrm{H} 7 \mathrm{a}), 2.30$ (dt, 1H, J 6.8, $1.6 \mathrm{~Hz}, \mathrm{H7b}$ ), 3.95-3.98 (m, 2H, H1, H4), 5.08 (d, 2H, J $12.2 \mathrm{~Hz}, \mathrm{H} 9 \mathrm{a}$ ), 
$5.11(\mathrm{~d}, 2 \mathrm{H}, \mathrm{J} 12.2 \mathrm{~Hz}, \mathrm{H} 9 \mathrm{~b}), 6.93$ (t, 2H, J2.0 Hz, H5, H6), 7.31-7.34 (m, 10H, $10 \times \mathrm{C} \underline{\mathrm{H}}) ;{ }^{13} \mathrm{C} \mathrm{NMR}\left(75 \mathrm{MHz}, \mathrm{CDCl}_{3}\right)$ $\delta 53.7$ (C1, C4), 66.7 (C9), 73.0 (C7), 128.2 (C13), 128.3 (C12, C14), 128.5 (C11, C15), 135.6 (C10), 142.4 (C5, C6), 152.3 (C2, C3), 165.0 (C8); HRMS (ESI) $\mathrm{m} / \mathrm{z}$, calcd. for $\mathrm{C}_{23} \mathrm{H}_{20} \mathrm{NaO}_{4}[\mathrm{M}+\mathrm{Na}]^{+}:$383.1254, found: 383.1246 .

Bis(2-methylbenzyl) bicyclo[2.2.1]hepta-2,5-diene2,3-dicarboxylate (14)

Yellow oil; yield 79\%; Rf $=0.22$ (hexane/ethyl acetate 3.9:0.1 v/v); IR (ATR) v / $\mathrm{cm}^{-1} 2940,1704,1621,1463$, 1224, 744; ${ }^{1} \mathrm{H}$ NMR $\left(200 \mathrm{MHz}, \mathrm{CDCl}_{3}\right) \delta 2.08-2.13(\mathrm{~m}, 1 \mathrm{H}$, H7a), 2.31-2.38 (m, 7H, H7b, H16), 3.95-3.98 (m, 2H, H1, H4), 5.07 (d, 2H, J $12.2 \mathrm{~Hz}, \mathrm{H} 9 \mathrm{a}), 5.12(\mathrm{~d}, 2 \mathrm{H}, J 12.2 \mathrm{~Hz}$, H9b), 6.93 (t, 2H, J 2.0 Hz, H5, H6), 7.17-7.28 (m, 8H, $\mathrm{H} 12, \mathrm{H} 13, \mathrm{H} 14, \mathrm{H} 15) ;{ }^{13} \mathrm{C} \mathrm{NMR}\left(50 \mathrm{MHz}, \mathrm{CDCl}_{3}\right) \delta 18.9$ (C16), 53.5 (C1, C4), 65.1 (C9), 72.9 (C7), 126.0 (C14), 128.5 (C13), 129.2 (C12), 130.3 (C15), 133.5 (C11), 137.0 (C10), 142.4 (C5, C6), 152.3 (C2, C3), 165.0 (C8); HRMS (ESI) $m / z$, calcd. for $\mathrm{C}_{25} \mathrm{H}_{24} \mathrm{NaO}_{4}[\mathrm{M}+\mathrm{Na}]^{+}$: 411.1567, found 411.1569 .

Bis(3-methylbenzyl) bicyclo[2.2.1]hepta-2,5-diene2,3-dicarboxylate (15)

Blue oil; yield 50\%; $\mathrm{Rf}=0.22$ (hexane/ethyl acetate 3.9:0.1 v/v); IR (ATR) v / cm $\mathrm{cm}^{-1} 2951,2877,1710,1626$, $1255,1232,781,692 ;{ }^{1} \mathrm{H}$ NMR $\left(300 \mathrm{MHz}, \mathrm{CDCl}_{3}\right) \delta 2.10$ (dt, $1 \mathrm{H}, J 6.8,1.6 \mathrm{~Hz}, \mathrm{H} 7 \mathrm{a}), 2.30(\mathrm{dt}, 1 \mathrm{H}, J 6.8,1.7 \mathrm{~Hz}$, H7b), 2.34 (s, 6H, $2 \times \mathrm{CH}_{3}$ ), 3.95-3.97 (m, 2H, H1, H4), 5.05 (d, 2H, J $12.2 \mathrm{~Hz}, \mathrm{H} 9 \mathrm{a}), 5.09$ (t, 2H, $J 12.2 \mathrm{~Hz}, \mathrm{H} 9 \mathrm{~b})$, 6.93 (d, 2H, J 2.0 Hz, H5, H6), 7.09-7.23 (m, 8H, $8 \times \mathrm{C} \underline{\mathrm{H}}$ ); ${ }^{13} \mathrm{C} \mathrm{NMR}\left(75 \mathrm{MHz}, \mathrm{CDCl}_{3}\right) \delta 21.3(\mathrm{C} 16), 53.5(\mathrm{C} 1, \mathrm{C} 4)$, 66.9 (C9), 73.0 (C7), 125.4 (C15), 128.4 (C14), 129.0 (C11, C13), 129.0 (C11), 135.4 (C10), 138.1 (C12), 142.4 (C5, C6), 152.4 (C2, C3), 165.0 (C8); HRMS (ESI) $\mathrm{m} / \mathrm{z}$, calcd. for $\mathrm{C}_{24} \mathrm{H}_{24} \mathrm{NaO}_{4}[\mathrm{M}+\mathrm{Na}]^{+}$: 411.1567, found: 411.1562 .

Bis(4-methylbenzyl) bicyclo[2.2.1]hepta-2,5-diene2,3-dicarboxylate (16)

White solid; yield $49 \%$; $\mathrm{mp} 75.0-75.8{ }^{\circ} \mathrm{C}$; $\mathrm{Rf}=0.22$ (hexane/ethyl acetate 3.9:0.1 v/v); IR (ATR) $\mathrm{v} / \mathrm{cm}^{-1} 2981$, 2953, 1702, 1612, 1269, 1051, 804, 727, 472; ${ }^{1} \mathrm{H}$ NMR (200 MHz, $\left.\mathrm{CDCl}_{3}\right) \delta 2.06-2.09$ (m, 1H, H7a), 2.26-2.35 (m, 7H, H7b, H16), 3.93-3.95 (m, 2H, H1, H4), 5.08 (d, 2H, $J 12.2 \mathrm{~Hz}, \mathrm{H} 9 \mathrm{a}), 5.11(\mathrm{~d}, 2 \mathrm{H}, J 12.2 \mathrm{~Hz}, \mathrm{H} 9 \mathrm{~b}), 6.93(\mathrm{t}, 2 \mathrm{H}$, $J$ 2.0 Hz, H5, H6), 7.12-7.23 (m, 8H, $8 \times \mathrm{C} \underline{\mathrm{H}}) ;{ }^{13} \mathrm{C} \mathrm{NMR}$ $\left(50 \mathrm{MHz}, \mathrm{CDCl}_{3}\right) \delta 21.2(\mathrm{C} 16), 53.5(\mathrm{C} 1, \mathrm{C} 4), 66.7$ (C9), 72.9 (C7), 128.5 (C13), 129.2 (C12, C14), 132.6 (C11, C15), 138.1 (C10), 142.4 (C5, C6), 152.2 (C2, C3), 165.0 (C8); HRMS (ESI) $\mathrm{m} / z$, calcd. for $\mathrm{C}_{25} \mathrm{H}_{24} \mathrm{NaO}_{4}[\mathrm{M}+\mathrm{Na}]^{+}$: 411.1567, found: 411.1569 .
Bis(3,5-dimethoxybenzyl) bicyclo[2.2.1]hepta-2,5-diene2,3-dicarboxylate (17)

Yellow oil; yield 22\%; Rf $=0.48$ (hexane/ethyl acetate 3.9:0.1 v/v); IR (ATR) v / $\mathrm{cm}^{-1} 2935,2835,1712,1597$, 1462, 1149, 835, 688; ${ }^{1} \mathrm{H}$ NMR $\left(200 \mathrm{MHz}, \mathrm{CDCl}_{3}\right)$ $\delta$ 2.08-2.12 (m, 1H, H7a), 2.28-2.32 (m, 1H, H7b), 3.76 (s, $12 \mathrm{H}, 4 \times \mathrm{C}_{3}$ ), 3.95-3.98 (m, 2H, H1, H4), 5.00-5.11 (m, 4H, H9), 6.38 (bs, 2H, H5, H6), 6.45-6.46 (m, 4H, $\mathrm{H} 11, \mathrm{H} 15), 6.91-6.93$ (m, 2H, H13); ${ }^{13} \mathrm{C}$ NMR (50 MHz, $\left.\mathrm{CDCl}_{3}\right) \delta 53.7$ (C1, C4), 55.4 (C16), 66.7 (C9), 73.1 (C7), 100.3 (C13), 106.0 (C11, C15), 137.9 (C10), 142.5 (C5, C6), 152.5 (C2, C3), 161.0 (C12, C14), 165.0 (C8); HRMS (ESI) $m / z$, calcd. for $\mathrm{C}_{27} \mathrm{H}_{28} \mathrm{NaO}_{8}[\mathrm{M}+\mathrm{Na}]^{+}$: 503.1676, found: 503.1676 .

Bis(4-fluorobenzyl) bicyclo[2.2.1]hepta-2,5-diene2,3-dicarboxylate (18)

White solid; yield 51\%; mp 70.3-71.7 ${ }^{\circ} \mathrm{C}$; Rf $=0.40$ (hexane/ethyl acetate 3.5:0.5 v/v); IR (ATR) $\mathrm{v} / \mathrm{cm}^{-1}$ 2998, 2950, 1702, 1600, 1512, 1272, 1220, 823; ' ${ }^{\mathrm{H}} \mathrm{NMR}$ $\left(300 \mathrm{MHz}, \mathrm{CDCl}_{3}\right) \delta 2.09$ (dt, 1H, J 6.8, $\left.1.5 \mathrm{~Hz}, \mathrm{H} 7 \mathrm{a}\right), 2.28$ (dt, 1H, J 6.8, $1.5 \mathrm{~Hz}, \mathrm{H} 7 \mathrm{~b}), 3.93-3.95$ (m, 2H, H1, H4), 5.04 (d, 2H, J 12.2 Hz, H9a), 5.08 (t, 2H, J $12.2 \mathrm{~Hz}, \mathrm{H} 9 \mathrm{~b}$ ), 6.91 (d, 2H, J 2.0 Hz, H5, H6), 6.97-7.03 (m, 4H, H11, H15), 7.24-7.29 (m, 4H, H12, H14); ${ }^{13} \mathrm{C}$ NMR (75 MHz, $\left.\mathrm{CDCl}_{3}\right) \delta 53.7$ (C1, C4), 66.1 (C9), $73.1(\mathrm{C} 7), 115.6(\mathrm{C} 12$, C14), 130.4 (C11, C15), 131.5 (C10), 142.5 (C5, C6), 152.5 (C2, C3), 162.8 (C13), 164.9 (C8); HRMS (ESI) $\mathrm{m} /$, calcd. for $\mathrm{C}_{23} \mathrm{H}_{18} \mathrm{~F}_{2} \mathrm{NaO}_{4}[\mathrm{M}+\mathrm{Na}]^{+}$: 419.1065, found: 419.1065 .

Bis(2-chlorobenzyl) bicyclo[2.2.1]hepta-2,5-diene2,3-dicarboxylate (19)

Yellow oil; yield 73\%; Rf $=0.48$ (hexane/ethyl acetate 3.9:0.1 v/v); IR (ATR) v / $\mathrm{cm}^{-1} 3064,2941,2869,1712$, 1620, 1250, 1224, 744, 707, 683; ' H NMR (300 MHz, $\left.\mathrm{CDCl}_{3}\right) \delta 2.12(\mathrm{dt}, 1 \mathrm{H}, J 6.9,1.5 \mathrm{~Hz}, \mathrm{H} 7 \mathrm{a}), 2.33(\mathrm{dt}, 1 \mathrm{H}$, $J$ 6.9, $1.7 \mathrm{~Hz}, \mathrm{H} 7 \mathrm{~b}$ ), 3.98-4.01 (m, 2H, H1, H4), 5.21 (d, 2H, J $12.2 \mathrm{~Hz}, \mathrm{H} 9 \mathrm{a}), 5.25$ (d, 2H, J $12.2 \mathrm{~Hz}, \mathrm{H} 9 \mathrm{~b}), 6.91$ (d, 2H, J 2.0 Hz, H5, H6), 7.19-7.26 (m, 4H, H15, H14), 7.30-7.36 (m, 4H, H12, H13); ${ }^{13} \mathrm{C} \mathrm{NMR}\left(75 \mathrm{MHz}, \mathrm{CDCl}_{3}\right.$ ) d 53.7 (C1, C4), 64.1 (C9), 72.9 (C7), 126.8 (C14), 129.4 (C13), 129.6 (C12), 133.2 (C11), 133.4 (C10), 142.5 (C5, C6), 152.4 (C2, C3), 164.6 (C8); HRMS (ESI) $\mathrm{m} / \mathrm{z}$, calcd. for $\mathrm{C}_{23} \mathrm{H}_{18} \mathrm{Cl}_{2} \mathrm{NaO}_{4}[\mathrm{M}+\mathrm{Na}]^{+}:$451.0474, found: 451.0472 .

Bis(4-chlorobenzyl) bicyclo[2.2.1]hepta-2,5-diene2,3-dicarboxylate (20)

Yellow solid; yield $56 \%$; $\mathrm{mp} 66.5-67.7^{\circ} \mathrm{C} ; \mathrm{Rf}=0.48$ (hexane/ethyl acetate 3.9:0.1 v/v); IR (ATR) $\mathrm{v} / \mathrm{cm}^{-1}$ 2999, 2944, 1700, 1616, 1491, 1267, 1093, 808, 721, 517; ${ }^{1} \mathrm{H}$ NMR $\left(200 \mathrm{MHz}, \mathrm{CDCl}_{3}\right) \delta$ 2.09-2.13 (m, 1H, H7a), 
2.28-2.32 (m, 1H, H7b), 3.97 (s, 2H, H1, H4), 5.10 (bs, 4H, H9a, H9b), 6.94 (bs, 2H, H5, H6), 7.15-7.33 (m, 8H, $8 \times \mathrm{CH}) ;{ }^{13} \mathrm{C}$ NMR $\left(50 \mathrm{MHz}, \mathrm{CDCl}_{3}\right) \delta 53.7(\mathrm{C} 1, \mathrm{C} 4)$, 66.0 (C9), 73.1 (C7), 128.8 (C12, C14), 129.7 (C11, C15), 134.1 (C13), 134.3 (C10), 142.5 (C5, C6), 152.5 (C2, C3), 164.9 (C8); HRMS (ESI) $\mathrm{m} / z$, calcd. for $\mathrm{C}_{23} \mathrm{H}_{18} \mathrm{Cl}_{2} \mathrm{NaO}_{4}$ $[\mathrm{M}+\mathrm{Na}]^{+}:$451.0474, found: 451.0483 .

Bis(2-bromobenzyl) bicyclo[2.2.1]hepta-2,5-diene2,3-dicarboxylate (21)

Colorless oil; yield 49\%; $\mathrm{Rf}=0.50$ (hexane/ethyl acetate 3.9:0.1 v/v); IR (ATR) $v / \mathrm{cm}^{-1} 3072,2949,2868,1714,1628$, $1234,1222,744,713,657 ;{ }^{1} \mathrm{H}$ NMR (300 MHz, $\left.\mathrm{CDCl}_{3}\right) \delta$ 2.13 (dt, $1 \mathrm{H}, J 6.8,1.5 \mathrm{~Hz}, \mathrm{H} 7 \mathrm{a}), 2.34$ (dt, $1 \mathrm{H}, J 6.8,1.5 \mathrm{~Hz}$, H7b), 4.00-4.02 (m, 2H, H1, H4), 5.18 (d, 2H, J $12.2 \mathrm{~Hz}$, H9a), 5.22 (d, $2 \mathrm{H}, J 12.2 \mathrm{~Hz}, \mathrm{H} 9 \mathrm{~b}), 6.94$ (t, $2 \mathrm{H}, J 2.0 \mathrm{~Hz}, \mathrm{H} 5$, H6), 7.15 (td, 2H, J 7.6, $1.9 \mathrm{~Hz}, \mathrm{H} 14$ ), 7.25 (td, 2H, J 7.6, $1.3 \mathrm{~Hz}, \mathrm{H} 13), 7.34$ (dd, 2H, J 7.6, 1.8 Hz, H15), 7.52 (dd, 2H, J 7.9, $1.3 \mathrm{~Hz}, \mathrm{H} 12) ;{ }^{13} \mathrm{C}$ NMR (75 MHz, $\left.\mathrm{CDCl}_{3}\right) \delta 53.7(\mathrm{C} 1$, C4), 66.2 (C9), 72.9 (C7), 123.3 (C11), 127.4 (C14), 129.6 (C12), 129.7 (C13), 132.7 (C15), 134.9 (C10), 142.4 (C5, C6), 152.3 (C2, C3), 164.6 (C8); HRMS (ESI) $\mathrm{m} / 2$, calcd. for $\mathrm{C}_{23} \mathrm{H}_{18} \mathrm{Br}_{2} \mathrm{NaO}_{4}[\mathrm{M}+\mathrm{Na}]^{+}$: 540.9445 , found: 540.9432 .

Bis(3-bromobenzyl) bicyclo[2.2.1]hepta-2,5-diene2,3-dicarboxylate (22)

White solid; yield 70\%; $\mathrm{mp} 40.4-41.9{ }^{\circ} \mathrm{C} ; \mathrm{Rf}=0.50$ (hexane/ethyl acetate 3.9:0.1 v/v); IR (ATR) $\mathrm{v} / \mathrm{cm}^{-1}$ 2996, 2943, 1704, 1612, 1571, 1263, 1049, 692; ${ }^{1} \mathrm{H}$ NMR $\left(200 \mathrm{MHz}, \mathrm{CDCl}_{3}\right) \delta 2.10-2.12(\mathrm{~m}, 1 \mathrm{H}, \mathrm{H} 7 \mathrm{a}), 2.30-2.32$ (m, 1H, H7b), 3.96-3.98 (m, 2H, H1, H4), 5.07 (d, 2H, $J 12.2 \mathrm{~Hz}, \mathrm{H} 9 \mathrm{a}), 5.10$ (d, 2H, J $12.2 \mathrm{~Hz}, \mathrm{H} 9 \mathrm{~b}), 6.93(\mathrm{t}, 2 \mathrm{H}$, $J 2.0$ Hz, H5, H6), 7.17-7.23 (m, 4H, H14, H15), 7.41-7.46 (m, 4H, H11, H13); $\left.{ }^{13} \mathrm{C} \mathrm{NMR} \mathrm{(50} \mathrm{MHz,} \mathrm{CDCl}_{3}\right) \delta 53.6(\mathrm{Cl}$, C4), 65.8 (C9), 73.0 (C7), 122.5 (C12), 126.6 (C15), 130.1 (C14), 131.1 (C13), 131.3 (C11), 137.7 (C10), 142.4 (C5, C6), 152.4 (C2, C3), 164.7 (C8); HRMS (ESI) $\mathrm{m} / z$, calcd. for $\mathrm{C}_{23} \mathrm{H}_{18} \mathrm{Br}_{2} \mathrm{NaO}_{4}[\mathrm{M}+\mathrm{Na}]^{+}$: 540.9445, found: 540.9442 .

Bis(4-bromobenzyl) bicyclo[2.2.1]hepta-2,5-diene2,3-dicarboxylate (23)

Yellow solid; yield $68 \%$; $\mathrm{mp} 92.7-94.0{ }^{\circ} \mathrm{C} ; \mathrm{Rf}=0.50$ (hexane/ethyl acetate 3.9:0.1 v/v); IR (ATR) v / $\mathrm{cm}^{-1} 2948$, 2877, 1693, 1608, 1518, 1050, 725; ${ }^{1} \mathrm{H}$ NMR $(200 \mathrm{MHz}$, $\left.\mathrm{CDCl}_{3}\right) \delta$ 2.07-2.12 (m, 1H, H7a), 2.26-2.31 (m, 1H, H7b), 3.93-3.97 (m, 2H, H1, H4), 5.02 (d, 2H, J $12.2 \mathrm{~Hz}, \mathrm{H} 9 \mathrm{a}$ ), 5.07 (d, 2H, J12.2 Hz, H9b), 6.93 (t, 2H, J 2.0 Hz, H5, H6), 7.13-7.17 (m, 4H, H11, H12), 7.41-7.48 (m, 4H, H13, H14); ${ }^{13} \mathrm{C} \mathrm{NMR}\left(50 \mathrm{MHz}, \mathrm{CDCl}_{3}\right) \delta 53.6(\mathrm{C} 1, \mathrm{C} 4), 65.9(\mathrm{C} 9), 73.0$ (C7), 122.3 (C12, C14), 129.8 (C11, C15), 131.7 (C13), 134.5 (C10), 142.4 (C5, C6), 152.4 (C2, C3), 164.7 (C8).
Bis(2-nitrobenzyl) bicyclo[2.2.1]hepta-2,5-diene2,3-dicarboxylate (24)

White solid; yield 89\%; $\mathrm{mp} 57.3-58.0{ }^{\circ} \mathrm{C}$; $\mathrm{Rf}=0.34$ (hexane/ethyl acetate 3.9:0.1 v/v); IR (ATR) $\mathrm{v} / \mathrm{cm}^{-1} 2979$, 2946, 2877, 1700, 1616, 1488, 1272, 1051, 802, 507; ${ }^{1} \mathrm{H}$ NMR $\left(200 \mathrm{MHz}, \mathrm{CDCl}_{3}\right) \delta$ 2.07-2.17 (m, 1H, H7a), 2.33-2.36 (m, 1H, H7b), 4.02 (s, 2H, H1, H4), 5.55 (bs, 4H, H9a, H9b), 6.93 (bs, 2H, H5, H6), 7.40-7.62 (m, 6H, H13, $\mathrm{H} 14, \mathrm{H} 15), 8.03-8.10$ (m, 2H, H12); ${ }^{13} \mathrm{C}$ NMR $(50 \mathrm{MHz}$, $\left.\mathrm{CDCl}_{3}\right) \delta 53.8(\mathrm{C} 1, \mathrm{C} 4), 63.5$ (C9), 73.1 (C7), 125.0 (C12), 128.8 (C13), 129.1 (C14), 131.6 (C15), 133.7 (C10), 142.5 (C5, C6), 147.4 (C11), 152.6 (C2, C3), 164.3 (C8); HRMS (ESI) $m / z$, calcd. for $\mathrm{C}_{23} \mathrm{H}_{18} \mathrm{~N}_{2} \mathrm{NaO}_{8}[\mathrm{M}+\mathrm{Na}]^{+}$: 473.0955 , found: 473.0959 .

Bis(3-nitrobenzyl) bicyclo[2.2.1] hepta-2,5-diene2,3-dicarboxylate (25)

Yellow solid; yield $80 \%$; $\mathrm{mp} 53.7-54.5^{\circ} \mathrm{C}$; $\mathrm{Rf}=0.34$ (hexane/ethyl acetate 3.9:0.1 v/v); IR (ATR) $\mathrm{v} / \mathrm{cm}^{-1} 3088$, 2939, 2870, 1704, 1618, 1524, 1520, 1232, 1070, 727; ${ }^{1} \mathrm{H}$ NMR $\left(200 \mathrm{MHz}, \mathrm{CDCl}_{3}\right) \delta$ 2.09-2.17 (m, 1H, H7a), 2.33-2.37 (m, 1H, H7b), 4.01-4.03 (m, 2H, H1, H4), 5.27 (bs, 4H, H9a, H9b), 6.96 (bs, 2H, H5, H6), 7.47-7.68 (m, $4 \mathrm{H}, \mathrm{H} 11, \mathrm{H} 13), 8.12-8.20$ (m, 4H, H14, H15); ${ }^{13} \mathrm{C}$ NMR (50 MHz, $\left.\mathrm{CDCl}_{3}\right) \delta 53.8$ (C1, C4), $65.3(\mathrm{C} 9), 73.1$ (C7), 122.7 (C13), 123.2 (C11), 129.5 (C14), 133.7 (C15), 137.6 (C10), 142.4 (C5, C6), 148.3 (C12), 152.8 (C2, C3), 164.3 (C8); HRMS (ESI) $\mathrm{m} / z$, calcd. for $\mathrm{C}_{23} \mathrm{H}_{18} \mathrm{~N}_{2} \mathrm{NaO}_{8}$ $[\mathrm{M}+\mathrm{Na}]^{+}:$473.0955, found: 473.0952 .

Synthesis of methyl ester $\mathbf{4}$

To a methanol $(35.0 \mathrm{~mL})$ solution of compound $\mathbf{3}$ $(0.180 \mathrm{~g}, 1.00 \mathrm{mmol})$ in a round bottomed flask was added concentrated sulfuric acid $(0.60 \mathrm{~mL})$ and the mixture was stirred at $25{ }^{\circ} \mathrm{C}$ for a period of $24 \mathrm{~h}$. The methanol was removed under reduced pressure and aqueous sodium bicarbonate $(45 \mathrm{~mL})$ was slowly added to neutralize the acidic residue. The aqueous mixture was transferred to a separating funnel and extracted with dichloromethane $(2 \times 30 \mathrm{~mL})$. The combined organic phases was dried with anhydrous magnesium sulfate, filtered, and the solvent was removed under reduced pressure to afford the desired compound (4) as a yellow oil in $98 \%$ yield.

Dimethyl bicyclo[2.2.1] hepta-2,5-diene-2,3-dicarboxylate (4)

Yellow oil; yield 98\%; $\mathrm{Rf}=0.76$ (hexane/ethyl acetate 1.5:0.5 v/v); IR (ATR) v / $\mathrm{cm}^{-1} 3007,2954,1704,1628$, 1437, 1324, 1326, 707; ' ${ }^{1} \mathrm{H}$ NMR (300 MHz, $\mathrm{CDCl}_{3}$ ) $\delta 2.09$ (dt, $1 \mathrm{H}, J 6.8,1.5 \mathrm{~Hz}, \mathrm{H} 7 \mathrm{a}), 2.27$ (dt, 1H, $J 6.8,1.6 \mathrm{~Hz}$, $\mathrm{H} 7 \mathrm{~b}), 3.77$ (s, 6H, $2 \times \mathrm{C}_{3}$ ), 3.92-3.94 (m, 2H, H1, H4), 
$6.91(\mathrm{t}, 2 \mathrm{H}, \mathrm{J} 2.0 \mathrm{~Hz}, \mathrm{H} 5, \mathrm{H} 6) ;{ }^{13} \mathrm{C} \mathrm{NMR}\left(75 \mathrm{MHz}, \mathrm{CDCl}_{3}\right)$ $\delta 52.1$ (C1, C4), 53.5 (C9), 73.0 (C7), 142.4 (C5, C6), 152.5 (C2, C3), 165.3 (C8); HRMS (ESI) $\mathrm{m} / \mathrm{z}$, calcd. for $\mathrm{C}_{11} \mathrm{H}_{12} \mathrm{NaO}_{4}[\mathrm{M}+\mathrm{Na}]^{+}: 231.0628$, found: 231.0628 .

\section{Evaluation of nematicidal activity}

Solanum lycopersicum (tomato Santa Clara cultivar) plants were used to obtain phytonematode Meloidogyne javanica via plant infestation. After 60 days of infestation, the plants were submitted to $M$. javanica eggs extraction. The eggs retained in a 500 mesh strainer were moved to a Baermann funnel, where it was incubated for $48 \mathrm{~h}$ to afford second stage $M$. javanica (J2) juveniles for use in the experiments.

The nematicidal activity of norbornadiene-derivatives was evaluated in 96-well flat bottomed plates (capacity of each well: $300 \mu \mathrm{L}$, Elisa-type plates). Each well represented an experimental plot and received $100 \mu \mathrm{L}$ of aqueous suspension, containing approximately 50 second stage (J2) juveniles and $100 \mu \mathrm{L}$ of each compound solution at the desired concentration. The nematicide tests were mounted on Elisa plates in a vertical laminar flow chamber to minimize possible sample contamination. After mounting, the plates were sealed with poly(vinyl chloride) (PVC) film and stored in a BOD chamber at $25^{\circ} \mathrm{C}$ in the absence of light.

The emulsifying agent Tween 80 aqueous solution $(10 \% \mathrm{~m} / \mathrm{v})$ was used to prepare the solutions of the synthesized compounds (3-25). The formulation of the synthesized compounds in Tween 80 was prepared at the concentration of $500 \mu \mathrm{g} \mathrm{mL} \mathrm{L}^{-1}$. The solution of each compound was transferred with the aid of a micropipette $(100 \mu \mathrm{L})$ to five wells in the Elisa-type plates. To each of these wells was added aqueous solution $(100 \mu \mathrm{L})$ containing approximately 50 nematodes. After these two steps the final concentration of each of the compounds was $250 \mu \mathrm{g} \mathrm{mL} L^{-1}$.

Distilled water and Tween 80 were kept in autoclave for $15 \mathrm{~min}$. Aqueous Tween $80(10 \%)$ and distilled water were used as negative controls. Aqueous solution of carbofuran (2,3-dihydro-2,2-dimethyl-1-benzofuran7-yl- $N$-methylcarbamate, 98\%, Sigma-Aldrich, St. Louis, United States) at concentration of $17.5 \mathrm{mg} \mathrm{mL}^{-1}$ was used as positive control.

The mortality was evaluated at 24,48 and $72 \mathrm{~h}$ after treatment application, using an Elisa plate for each day of the evaluation. For the evaluation, $5 \mu \mathrm{L}$ of a freshly prepared aqueous $\mathrm{NaOH}$ solution $\left(1.0 \mathrm{~mol} \mathrm{~L} \mathrm{~L}^{-1}\right)$ was added to each well and then the number of live and dead nematodes was counted. Nematodes that changed their body shape within
1 min were considered alive, while nematodes that did not react to the addition of $\mathrm{NaOH}$ were considered dead. The values were transformed in percentage before the statistical analysis. This experiment was performed in a random design with five replications for each treatment. The test was performed twice to confirm the results.

The compounds $\mathbf{3}$ and $\mathbf{4}$ that presented nematicidal potential were used to determine the lethal concentration that kills $50 \%\left(\mathrm{LD}_{50}\right)$ of the nematodes. Compounds 3 and 4 at the concentration of 15.6, 31.2, 62.5, 125 and $250 \mu \mathrm{g} \mathrm{mL}^{-1}$ were evaluated against Meloidogyne javanica. At this experiment the mortality of the nematodes was visualized at $48 \mathrm{~h}$ of incubation (period in which the substances presented the best mortality results), following the mounting and evaluation methodology described above. An Elisa plate was used for each compound with its appropriate concentration. Each bioassay was replicated five times in a completely randomized design. With the mortality results, a mortality versus concentration graph was plotted, where the curve that best represents the results was obtained for each compound. After obtaining them, the interpolation was performed obtaining the $\mathrm{LD}_{50}$ for substances $\mathbf{3}$ and $\mathbf{4}$.

\section{Statistical analysis}

Initially, data normality and homogeneity were evaluated by the Shapiro-Wilk and Bartlett tests, showing positive results for both homogeneity and normality, indicating that the data are parametric. The values were subjected to variance analysis and means were compared by the Scott-Knott test at probability of $\alpha=0.05$ level. Statistical analysis together with mortality values is shown in Table S1 of the Supplementary Information (SI) section.

\section{Results and Discussion}

\section{Synthesis}

Diels-Alder reaction of acetylenedicarboxylic acid monopotassium salt (potassium 3-carboxypropiolate) with cyclopentadiene afforded bicyclo[2.2.1]hepta-2,5-diene2,3-dicarboxylic acid (3). ${ }^{26}$

Bimolecular nucleophilic substitution reactions $\left(\mathrm{S}_{\mathrm{N}} 2\right)$ of alkyl and benzyl bromides with the dicarboxylate derived from compound 3 provided 21 esters (5-25) in yields that varied from 22 to $89 \%$ after silica-gel column chromatography (Scheme 1). The dimethyl ester (4) was obtained by esterification with methanol catalyzed by concentrated sulfuric acid. The reaction mixture was neutralized with potassium carbonate and extracted with 
dichloromethane to provide the dimethyl ester in $98 \%$ yield. All compounds were fully characterized by extensive spectrometric analyses.

The infrared spectrum of the dicarboxylic acid (3) presented a characteristic band of carbonyl stretching at $1693 \mathrm{~cm}^{-1}$ and the diester derivatives (4-25) presented this band at ca. $1700 \mathrm{~cm}^{-1}$. The diastereotopic bridge head hydrogens $\mathrm{H} 7 \mathrm{a}$ and $\mathrm{H} 7 \mathrm{~b}$ of the diesters were shown as doublet of triplets in the ${ }^{1} \mathrm{H}$ NMR. The multiplet at $\delta$ ca. $0.9 \mathrm{ppm}$ was assigned to the methyl groups (diesters 7-12). This multiplicity is explained by the virtual coupling to the methylene groups (more than four $\mathrm{CH}_{2}$ groups) at $\delta$ ca. $1.3 \mathrm{ppm}$. The signals at $\delta 165,152$, and $142 \mathrm{ppm}$ in the ${ }^{13} \mathrm{C}$ NMR were assigned to the carbonyl groups, alkene carbons $\mathrm{C} 2 / \mathrm{C} 3$ (non-hydrogenated) and C5/C6 $(=\mathrm{CH})$, respectively. The electron withdrawing effect of the carbonyl groups deshields carbons C2/C3 compared to C5/C6, which are more shielded and larger than $\mathrm{C} 2 / \mathrm{C} 3$ because of the nuclear Overhauser effect (NOE). Electrospray high resolution mass spectra of these compounds confirmed the proposed structures. The spectra of the synthesized compounds can be found in the SI section.

\section{Biological assay}

Compounds 3-25 at the concentration of $250 \mu \mathrm{g} \mathrm{mL}^{-1}$ were submitted to biological evaluation against $M$. javanica, the mortality was verified at 24,48 , and $72 \mathrm{~h}$ after contact of the nematodes with the aqueous solution of the compounds. The results were plotted as a bar graph to visualize the potential of the compounds evaluated to kill nematodes (Figure 1).

The nematocidal potential was observed $24 \mathrm{~h}$ after nematode contact with the aqueous solution of the compounds under study. A higher potential was observed at $48 \mathrm{~h}$ and no activity increase was observed after a longer period of nematode contact with the aqueous solution of the compounds.

Compounds 3 and $\mathbf{4}$ caused more than 90\% mortality of the nematodes at $48 \mathrm{~h}$, and compounds 5-25 caused

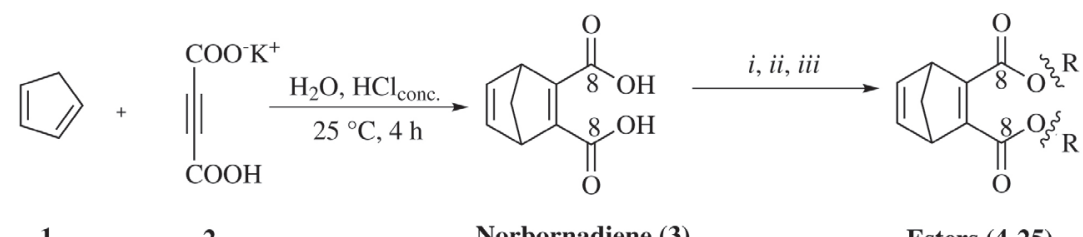

1

2

Norbornadiene (3)

Esters (4-25)

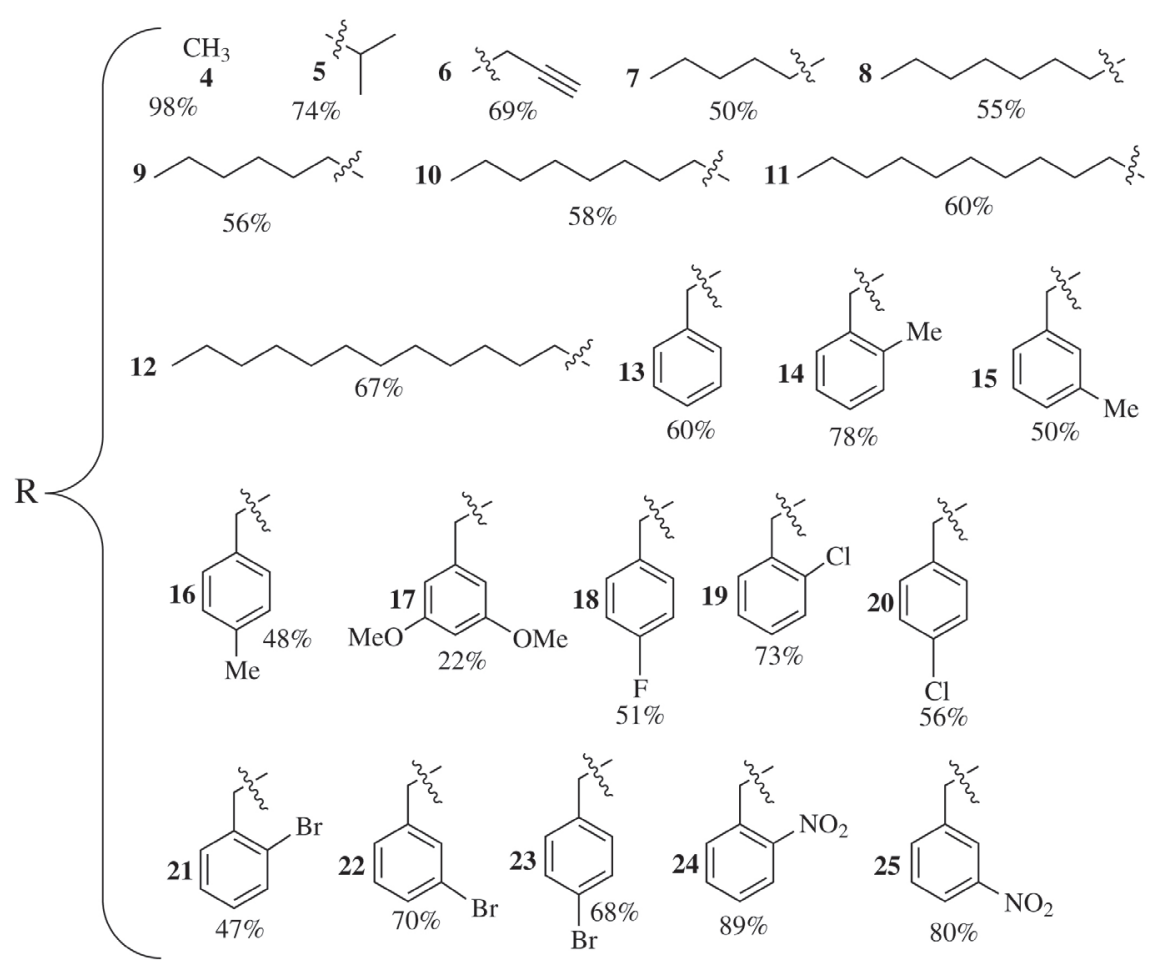

Scheme 1. Syntheses of the esters 4-25 via Diels-Alder adduct reaction. (i) $\mathrm{CH}_{3} \mathrm{OH}, \mathrm{H}_{2} \mathrm{SO}_{4}, 24 \mathrm{~h}$; (ii) DMA, $\mathrm{K}_{2} \mathrm{CO}_{3}, 70{ }^{\circ} \mathrm{C}$, alkyl bromide, $18 \mathrm{~h}(\mathrm{esters}$ 4-12); (iii) DMA, $\mathrm{K}_{2} \mathrm{CO}_{3}, 25^{\circ} \mathrm{C}$, benzyl bromide, $18 \mathrm{~h}$ (esters 13-25). 

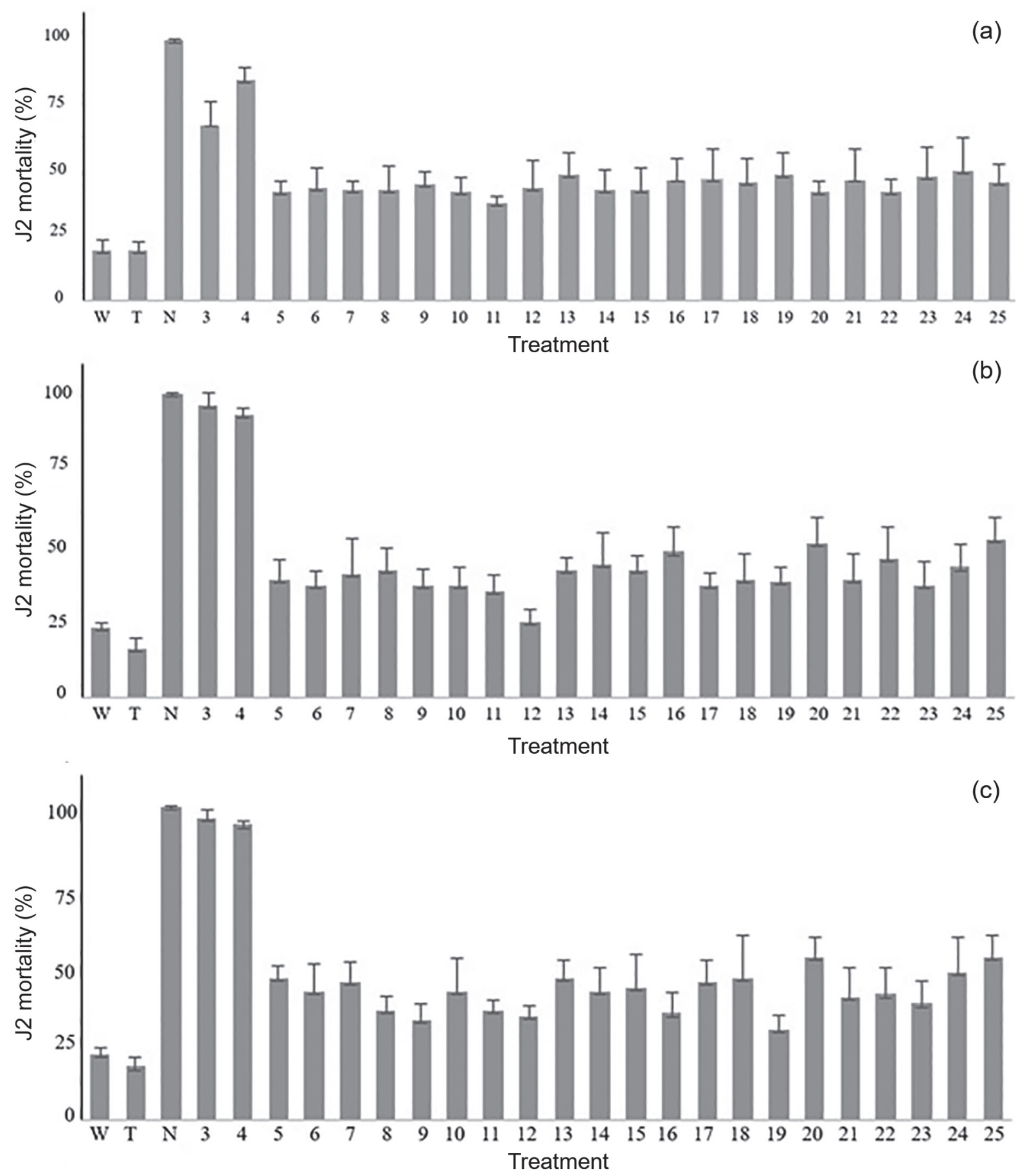

Figure 1. Mortality of second stage juveniles (J2) versus norbornadiene derived compounds. (a) Percentage of mortality assessed at 24 h; (b) percentage of mortality assessed at $48 \mathrm{~h}$; (c) percentage of mortality assessed at $72 \mathrm{~h}$. W = water; $\mathrm{T}=$ tween $80(10 \%) ; \mathrm{N}=$ nematicide carbofuran.

approximately $50 \%$ mortality at this same time period. Nematode mortality was not increased at $72 \mathrm{~h}$. Therefore, maximum of mortality was reached with $48 \mathrm{~h}$. Thus, a shorter period of action of the compounds can be considered a positive feature, especially for controlling nematode infestation to prepare the soil to grow a crop.

Dicarboxylic acid $\mathbf{3}$ caused $96 \%$ mortality of nematodes and the dimethyl ester 4 killed $93 \%$ at $48 \mathrm{~h}$. The more lipophilic dialkyl and dibenzyl esters (5-25) have killed between 30 and $52 \%$ of nematodes. The overall nematicide activities of these compounds may be related to their relative polarities, because the most polar dicarboxylic acid 3 and dimethyl ester $\mathbf{4}$ presented the best results compared to the most lipophilic esters (5-25). ${ }^{23}$

Lethal concentration to kill $50 \%$ of the nematodes $\left(\operatorname{LD}_{50}\right)$ was determined for the most active compounds $\mathbf{3}$ and $\mathbf{4}$ (final 
concentration in the well: 250, 125, 62, 31, $16 \mu \mathrm{g} \mathrm{mL}^{-1}$ ). This experiment was carried out as described for the assay at the concentration of $250 \mu \mathrm{g} \mathrm{mL}-1$. The $\mathrm{LD}_{50}$ determined for compound 3 was $11.8 \mu \mathrm{g} \mathrm{mL}^{-1}$ and for compound $\mathbf{4}$ was $99.4 \mu \mathrm{g} \mathrm{mL}{ }^{-1}$. The nematocidal potential of compound $\mathbf{3}$ is greater than that of compound $\mathbf{4}$ as the $\mathrm{LD}_{50}$ for compound $\mathbf{3}$ is 9 times smaller. These results are better than those obtained using essential oils ${ }^{27}$ with assays on $M$. javanica that showed $\mathrm{LD}_{50}$ greater than $200 \mu \mathrm{g} \mathrm{mL} \mathrm{g}^{-1}$. Aliphatic esters were also tested against pine wood nematode B. xylophilus ${ }^{28}$ and the values ranged from 21 to $220 \mu \mathrm{g} \mathrm{mL}^{-1}$.

Compound $\mathbf{3}$ was obtained in high yields in one step from readily available starting materials, namely cyclopentadiene and acetylenedicarboxylic acid monopotassium salt. The reaction was carried out in aqueous medium and the product was isolated by solvent extraction and purified by hexane washings. Considering the in vitro nematocidal results so far obtained, and the easy synthetic access, it is clear that the dicarboxylic acid $\mathbf{3}$ presents potential to be used in the development of new products to control M. javanica. Although the mechanism of action for this series of compounds against $M$. javanica has not been under scrutiny, the in vitro results presented in this work suggest that polarity of the compounds is an important feature.

\section{Conclusions}

In this work, bicyclo[2.2.1]hepta-2,5-diene2,3-dicarboxylic acid (3) and alkyl and aryl esters were synthesized in moderate to high overall yields. After evaluation of the nematocidal activity against $M$. javanica, we observed that the dicarboxylic acid (3) and the dimethyl ester (4) derivatives exhibited better activities than the dialkyl and diaryl esters (5-25). The greater nematocidal activities of compounds $\mathbf{3}$ and $\mathbf{4}$ than the more lipophilic dialkyl and diaryl esters suggest that the polarity of the compounds is a key feature. Considering the easy synthetic access for these compounds, in vivo bioassays are under consideration as a next step, to further evaluate the potential nematocidal activity of these compounds in tomato crops.

\section{Supplementary Information}

Supplementary information $\left({ }^{1} \mathrm{H},{ }^{13} \mathrm{C}\right.$ and IR spectra, Tables S1 and S2) is available free of charge at http://jbcs.sbq.org.br as PDF file.

\section{Acknowledgments}

We thank the following for grant and scholarship financial support: the Fundação de Amparo à Pesquisa do Estado de
Minas Gerais (FAPEMIG) and the Conselho Nacional de Desenvolvimento Científico e Tecnológico (CNPq) (A. J. D., E. S. A.), and Coordenação de Aperfeiçoamento de Pessoal de Nível Superior (CAPES, finance code 001) and fellowship (A. C. S. G., A. R. F., J. P. E. G.).

\section{Author Contributions}

A. C. S. G., A. J. D. and E. S. A. performed the experimental work of synthesis and characterization of compounds, wrote and reviewed the manuscript; A. C. S. G., J. P. E. G., D. S. B., A. R. F. performed the nematicidal assays, analyzed the results and wrote part of this manuscript; E. J. P., E. S. performed the HRMS spectra, analyzed the HRMS data and reviewed the manuscript.

\section{References}

1. Xu, X. B.; Liu, Y. N.; Rao, G. W.; Russ. J. Org. Chem. 2019 , 55, 559.

2. Wolke, C.; Daniliuc, C. G.; Kehr, G.; Erker, G.; J. Organomet. Chem. 2019, 899, 120879.

3. Levandowski, B. J.; Svatunek, D.; Sohr, B.; Mikula, H.; Houk, K. N.; J. Am. Chem. Soc. 2019, 141, 2224.

4. Kim, H.; Gerosa, G.; Aronow, J.; Kasaplar, P.; Ouyang, J.; Lingnau, J. B.; Guerry, P.; Fares, C.; List, B.; Nat. Commun. 2019, 10, 770 .

5. Salles, R. C.; Lacerda, V.; Barbosa, L. R.; Ito, F. M.; Lima, D. P.; Santos, R. B.; Greco, S. J.; Neto, A. C.; Castro, E. V. R.; Beatriz, A.; J. Mol. Struct. 2012, 1007, 191.

6. Geldenhuys, W. J.; Malan, S. F.; Bloomquist, J. R.; Marchand, A. P.; Van Der Schyf, C. J.; Med. Res. Rev. 2005, 25, 21.

7. Abraham, I.; Joshi, R.; Pardasani, P.; Pardasani, R. T.; J. Braz. Chem. Soc. 2011, 22, 385.

8. da Silva, F. C.; Ferreira, S. B.; Kaiser, C. R.; Pinto, A. C.; Ferreira, V. F.; J. Braz. Chem. Soc. 2009, 20, 1478.

9. Gültekin, D. D.; Taşkesenligil, Y.; Daştan, A.; Balci, M.; Tetrahedron 2008, 64, 4377.

10. Nişanci, B.; Dalkilic, E.; Güney, M.; Dastan, A.; J. Org. Chem. 2009, $5,4$.

11. Spivack, K. J.; Walker, J. V.; Sanford, M. J.; Rupert, B. R.; Ehle, A. R.; Tocyloski, J. M.; Jahn, A. N.; Shaak, L. M.; Obianyo, O.; Usher, K. M.; Goodson, F. E.; J. Org. Chem. 2017, 82, 1301.

12. Brummel, O.; Waidhas, F.; Bauer, U.; Wu, Y.; Bochmann, S.; Steinrück, H. P.; Papp, C.; Bachmann, J.; Libuda, J.; J. Phys. Chem. Lett. 2017, 8, 2819.

13. Quant, M.; Lennartson, A.; Dreos, A.; Kuisma, M.; Erhart, P.; Börjesson, K.; Moth-Poulsen, K.; Chem. - Eur. J. 2016, 22, 13265.

14. Manso, M.; Petersen, A. U.; Wang, Z.; Erhart, P.; Nielsen, M. B.; Moth-Poulsen, K.; Nat. Commun. 2018, 9, 1945. 
15. Sabirov, D. S.; Terentyev, A. O.; Shepelevich, I. S.; Bulgakov, R. G.; Comput. Theor. Chem. 2014, 1045, 86.

16. Serhan, Z.; Aroulanda, C.; Lesot, P.; J. Phys. Chem. A 2016, $120,6076$.

17. Tebikachew, B. E.; Li, H. B.; Pirrotta, A.; Börjesson, K.; Solomon, G. C.; Hihath, J.; Moth-Poulsen, K.; J. Phys. Chem. C 2017, 121, 7094.

18. Akker, S. E.; Laetsch, D. R.; Thorpe, P.; Lilley, C. J.; Danchin, E. G. J.; Rocha, M.; Rancurel, C.; Holroyd, N. E.; Cotton, J. A.; Szitenberg, A.; Grenier, E.; Montarry, J.; Mimee, B.; Duceppe, M. O.; Boyes, I.; Marvin, J. M. C.; Jones, L. M.; Yusup, H. B.; Lafond-Lapalme, J.; Esquibet, M.; Sabeh, M.; Rott, M.; Overmars, H.; Finkers-Tomczak, A.; Smant, G.; Koutsovoulos, G.; Blok, V.; Mantelin, S.; Cock, P. J. A.; Phillips, W.; Henrissat, B.; Urwin, P. E.; Blaxter, M.; Jones, J. T.; Genome Biol. 2016, 17,124 .

19. Ibrahim, H. M. M.; Ahmad, E. M.; Martínez-Medina, A.; Aly, M. A. M.; Plant Physiol. Biochem. 2019, 141, 332.

20. Talwana, H.; Sibanda, Z.; Wanjohi, W.; Kimenju, W.; Luambano-Nyoni, N.; Massawe, C.; Manzanilla-López, R. H.; Davies, K. G.; Hunt, D. J.; Sikora, R. A.; Coyne, D. L.; Gowen, S. R.; Kerry, B. R.; Pest Manage. Sci. 2016, 72, 226.
21. Ebone, L. A.; Kovaleski, M.; Deuner, C.; Plant Sci. Today 2019, 6,91 .

22. Moreno, S. C.; Silverio, F. O.; Picanco, M. C.; Alvarenga, E. S.; Pereira, R. R.; Santana Jr., P. A.; Silva, G. A.; J. Insect Sci. 2017, 17, 6 .

23. Teixeira, M. G.; Alvarenga, E. S.; Lopes, D. T.; Oliveira, D. F.; Pest Manage. Sci. 2019, 75, 3331.

24. Lopes, M.; Alvarenga, E.; Aguiar, A.; Santos, I.; Silva, G.; Arcanjo, L.; Picanco, M.; Quim. Nova 2018, 41, 375.

25. Oliveira, D. F.; Costa, V. A.; Terra, W. C.; Campos, V. P.; Paula, P. M.; Martins, S. J.; Exp. Parasitol. 2019, 199, 17.

26. Carman, R. M.; Derbyshire, R. P. C.; Hansford, K. A.; Kadirvelraj, R.; Robinson, W. T.; Aust. J. Chem. 2001, 54, 117.

27. Andres, M. F.; Gonzalez-Coloma, A.; Sanz, J.; Burillo, J.; Sainz, P.; Phytochem. Rev. 2012, 11, 371.

28. Seo, S. M.; Kim, S. H.; Ahn, Y. J.; Park, I.; J. Agric. Food Chem. 2014, 62, 9103.

Submitted: December 27, 2019

Published online: April 9, 2020 